\title{
Actividades Prácticas en Anatomía: Factor Nivelador de los Contrastes de Origen entre los Alumnos de Medicina
}

\author{
Practical Activities in Anatomy: Leveling Factor for the Origin \\ Related Contrasts Among Medical Students
}

Oscar Inzunza ${ }^{1}$; Daniela Schneeberger ${ }^{1}$; Guillermo Salgado ${ }^{1} \&$ Mario Cantín $^{2 \dagger}$

INZUNZA, O.; SCHNEEBERGER, D.; SALGADO, G. \& CANTÍN, M. Actividades prácticas en anatomía: factor nivelador de los contrastes de origen entre los alumnos de medicina. Int. J. Morphol., 35(2):494-499, 2017.

RESUMEN: Las mediciones de los logros educacionales y las pruebas de selección de ingreso a la educación superior están fuertemente correlacionadas con los antecedentes socioeconómicos y el tipo de establecimiento educacional del postulante, situación que representaría un factor predictor del rendimiento para los alumnos de primer año universitario. Considerando que los puntajes de ingreso para la carrera de medicina presentan una diferencia significativa entre los primeros y últimos seleccionados, nos preguntamos si existe algún factor nivelador que reduzca esa brecha de origen. Se analiza el rendimiento en las evaluaciones prácticas y pruebas teóricas, al comienzo y al final del año académico 2009 comparando los primeros y últimos seleccionados de acuerdo al puntaje de ingreso PSU a la carrera de medicina de la Pontificia Universidad Católica de Chile. Se observan diferencias significativas en el rendimiento de la primera evaluación Teórica de Anatomía y en la Nota Final obtenida en el curso, en cambio, no se encuentran diferencias significativas tanto en la primera Evaluación Práctica cómo en el examen final práctico del curso. Nuestros resultados sugieren que la única evaluación niveladora, que logra superar las diferencias de origen de los examinados, la constituyen nuestras pruebas prácticas; evaluaciones en las cuales el rendimiento de los primeros y de los últimos seleccionados de medicina es más homogéneo. Este tipo de evaluaciones prácticas, realizadas sobre preparaciones cadavéricas, demandan fundamentalmente experiencias vividas en nuestras actividades prácticas, colocándose por sobre la mayor o menor habilidad o experiencia que posea el estudiante relacionada con su establecimiento educacional de origen.

PALABRAS CLAVE: Educación médica; Estudiantes de medicina; Aprendizaje médico; Ecoe; Osce.

\section{INTRODUCCIÓN}

Dadas las características de los planes y programas de la enseñanza media hasta la década de los 70, las universidades chilenas recibían una población de estudiantes que poseía un importante capital cultural expresado en el manejo de diversas competencias académicas (Scharager \& Sebastián, 2007), un adecuado manejo del lenguaje y una gama de experiencias vitales "suficientemente amplias" (conocimiento de otras culturas y de los artistas clásicos), que capacitaban al alumno para dialogar coloquialmente con los docentes encargados de su formación superior (Northedge, 2003).

A diferencia de lo que ocurrió durante gran parte del siglo XX, los estudiantes que hoy acceden a la educación superior presentan una gran variabilidad curricular e individual (Zúñiga et al., 2009) y una diversidad en su capital cultural inicial, que refleja diferencias de género, origen étnico, origen (sub)cultural, recursos económicos (del estudiante y su grupo familiar), nacionalidad, distintas formas de discapacidad y competencias de bases cognitivas, afectivas y sociales (Northedge; Barrington, 2004)

Así, como señala Mónica Chacón “De la educación pública que tradicionalmente permitió el surgimiento de grandes liderazgos sociales, políticos y culturales en nuestro país, hoy no queda más que el recuerdo"; porqué, a partir de los años ochenta, la educación ha estado sujeta a la lógica del libre mercado, situación que sin duda ha extremado los problemas de acceso a la educación, colocándola al servicio de valores que priorizan el individualismo y la competencia (Chacón, 2003).

\footnotetext{
${ }^{1}$ Departamento de Anatomía, Escuela de Medicina, Pontificia Universidad Católica de Chile, Chile.

${ }^{2 \dagger}$ Programa de Doctorado en Ciencias Morfológicas, Facultad de Odontología, Universidad de la Frontera.
} 
En efecto, a partir de esa década, el ministerio de educación delegó en manos de corporaciones municipales y fundaciones privadas la gestión administrativa, financiera y curricular de escuelas y liceos; desencadenándose un proceso privatizador que incrementó los aportes económicos a colegios particulares subvencionados y redujo recursos a las escuelas fiscales municipalizadas. A inicios de los años 80 una cuarta parte de los colegios pertenecían a la esfera particular, en 1999 más del doble se encontraba en manos privadas, situación que afecta notoriamente la equidad en el acceso a la educación (Chacón).

Desde 2003 se emplean como factores de selección los resultados de una batería de pruebas de selección universitaria (PSU) y el promedio de notas de enseñanza media (NEM) de los postulantes. Esta PSU posee ciertas características que no favorecen ni garantizan la existencia de perfiles de ingresos reales y operativos sino que, dependiendo de cada carrera y universidad, estos factores se ponderan bajo el supuesto de que permiten predecir el éxito académico de los alumnos durante su estudio universitario (Contreras et al., 2009).

Es un hecho innegable que las mediciones de los logros educacionales y las pruebas de selección de ingreso a la educación superior están fuertemente correlacionadas con ese capital cultural inicial. Este capital es tan diverso como individuos existan, lo que sería un factor predictor del rendimiento académico de los alumnos durante el primer año universitario (Rocha et al., 2009; Inzunza, 2014).

Al observar la oscilación de más de 55 puntos en los puntajes de ingreso para la carrera de medicina año 2009, nos preguntamos, si esa diferencia numérica es significativa entre los alumnos de más "bajo" y más "alto" rendimien- to en la PSU y, de ser así, si esa diferencia cuantitativa se mantiene una vez terminado el año lectivo, o bien si existe algún factor que a lo largo del curso anual de Anatomía Normal que logre nivelar estas diferencias.

\section{MATERIAL Y MÉTODO}

Nuestro universo (U) correspondió a 90 alumnos de primer año de la carrera de medicina de la Pontificia Universidad Católica de Chile año 2009, vía de ingreso PSU, de los cuales se escoge una muestra correspondiente a los 10 primeros (AM) y 10 últimos (BM) alumnos seleccionados deacuerdo al puntaje de ingreso PSU. ( $\mathrm{n}=20,16,7 \% /$ U), separándolos en dos grupos de acuerdo a este criterio.

Criterio de exclusión para la muestra: Alumnos que hubiesen cursado alguna carrera universitaria.

Una vez realizada la separación de los individuos de cada grupo muestral (AM y BM), se tabularon los datos referidos al puntaje de ingreso (PI), nota obtenida en las evaluaciones prácticas (gymkanas) inicial (GYMI) y en el examen práctico final del curso (EXPF), evaluación teórica global inicial (GLOBI), y por último la nota final (NF) del año académico 2009 (Tabla I).

Posterior a ello se realizó el análisis estadístico descriptivo e inferencial de los datos obtenidos, mediante el uso del software Sigma Plot 10.0 para Windows 7, dónde se confrontaron ambos grupos (AM y BM) en las variables de puntaje de ingreso, y notas de gymkana inicial, examen práctico final, prueba global inicial y nota final del curso.

Tabla I. Notas obtenidas en Gymkana Inicial (GYMI), Examen práctico Final (EXPF), Prueba Global Inicial (GLOBI) y Nota Final (NF) por grupo muestral, de acuerdo a puntaje de ingreso (PI) obtenido en la PSU 2009.

\begin{tabular}{llllllllllll}
\hline $\mathbf{A M}$ & $\mathbf{P I}$ & GYMI & $\mathbf{E X P F}$ & GLOBI & $\mathbf{N F}$ & $\mathbf{B M}$ & $\mathbf{P I}$ & $\mathbf{G Y M I}$ & EXPF & GLOBI & NF \\
\hline $\mathbf{1}$ & 837,70 & 5,72 & 6,50 & 6,61 & 6,49 & $\mathbf{1}$ & 784,50 & 6,59 & 6,21 & 6,28 & 6,54 \\
$\mathbf{2}$ & 828,20 & 6,19 & 6,29 & 5,23 & 6,00 & $\mathbf{2}$ & 783,10 & 5,31 & 4,58 & 3,54 & 4,61 \\
$\mathbf{3}$ & 823,50 & 6,30 & 6,66 & 6,11 & 6,51 & $\mathbf{3}$ & 783,00 & 3,73 & 4,87 & 2,68 \\
$\mathbf{4}$ & 823,20 & 6,54 & 6,29 & 5,86 & 6,17 & $\mathbf{4}$ & 782,80 & 5,84 & 4,46 & 5,47 & 4,48 \\
$\mathbf{5}$ & 820,75 & 6,77 & 6,54 & 6,27 & 6,47 & $\mathbf{5}$ & 782,70 & 5,72 & 6,75 & 5,59 & 6,78 \\
$\mathbf{6}$ & 819,45 & 5,43 & 6,33 & 6,16 & 6,05 & $\mathbf{6}$ & 782,10 & 4,96 & 4,66 & 4,71 & 5,27 \\
$\mathbf{7}$ & 817,05 & 4,90 & 5,83 & 4,18 & 5,36 & $\mathbf{7}$ & 781,30 & 6,36 & 6,37 & 5,32 \\
$\mathbf{8}$ & 816,75 & 6,13 & 5,58 & 5,97 & 5,90 & $\mathbf{8}$ & 781,25 & 6,30 & 6,00 & 4,77 & 6,18 \\
$\mathbf{9}$ & 814,25 & 5,78 & 6,29 & 5,70 & 5,89 & $\mathbf{9}$ & 780,80 & 5,25 & 6,00 & 5,35 & 5,60 \\
$\mathbf{1 0}$ & 810,75 & 5,02 & 6,29 & 5,84 & 5,86 & $\mathbf{1 0}$ & 780,65 & 5,19 & 6,33 & 5,44 & 5,67
\end{tabular}

AM: Primeros seleccionados; BM: Últimos seleccionados; PI: Puntaje de Ingreso; GYMI: Gymkana Inicial; EXPF: Examen práctico Final; GLOBI: Prueba Global Inicial; NF: Nota Final del curso. 


\section{RESULTADOS}

Al realizar el análisis estadístico descriptivo de la muestra (n) de los puntajes de ingreso de ambos grupos tenemos que, el promedio y la mediana para el grupo de alto puntaje son de 821,16 y 820,1 puntos respectivamente, mientras que para el grupo de bajo puntaje son de 782,22 y 782,4 puntos. Al aplicarle la prueba no paramétrica de Mann-
Whitney Rank Sum Test, el resultado arroja diferencias claramente significativas entre ambos grupos con un índice de confianza de un $99 \%$ dando un valor $\mathrm{P}<0,001$. Esto nos permite asegurar que tenemos grupos heterogéneos de ingreso lo cual aprueba el posterior análisis de las otras variables. (Fig. 1, Tabla II)

Tabla II. Análisis estadístico de la distribución de los datos observados en el gráfico 1, con Mann-Whitney Rank Sum Test, observándose diferencias estadísticamente significativas entre ambos grupos.

\begin{tabular}{llllll}
\hline Group Name & $\mathbf{n}$ & Missing & Median & $\mathbf{2 5} \%$ & $\mathbf{7 5 \%}$ \\
\hline AM-PI & 10 & 0 & 820,1 & 816,75 & 823,5 \\
BM-PI & 10 & 0 & 782,4 & 781,25 & 783 \\
Mann-Whitney & U Statistic & $T=155,000$ & $n$ (small) $=10$ & $n($ big $)=10$ & \\
\hline
\end{tabular}

Tabla III. Correlación estadística de ambos grupos muestrales para la distribución de los datos observados en el gráfico 2, con t-test y Mann-Whitney Rank Sum Test para la Gymkana Inicial y Examen práctico final respectivamente.

\begin{tabular}{llllll}
\hline Group Name & $\mathbf{n}$ & Missing & Mean & Std Dev & SEM \\
AM-GYMI & 10 & 0 & 5,878 & 0,624 & 0,197 \\
BM-GYMI & 10 & 0 & 5,525 & 0,84 & 0,266 \\
t-test & $t=1,067$ & with 18 degrees & of freedom. & & \\
\hline Group Name & $\mathbf{n}$ & Missing & Median & $\mathbf{2 5 \%}$ & $\mathbf{7 5 \%}$ \\
\hline AM-EXPF & 10 & 0 & 6,290 & 6,290 & 6,500 \\
BM-EXPF & 10 & 0 & 6,000 & 4,660 & 6,330 \\
Mann-Whitney U Statistic & $T=125,500$ & $n($ small $)=10$ & $n($ big $)=10$ & &
\end{tabular}

$\overline{(P}=<0,05)$ AM-GYMI: Gymkana Inicial primeros seleccionados; BM-GYMI: Gymkana Inicial últimos seleccionados; AM-EXPF: Examen Práctico Final primeros seleccionados: BM- EXPF: Examen Práctico Final últimos seleccionados.
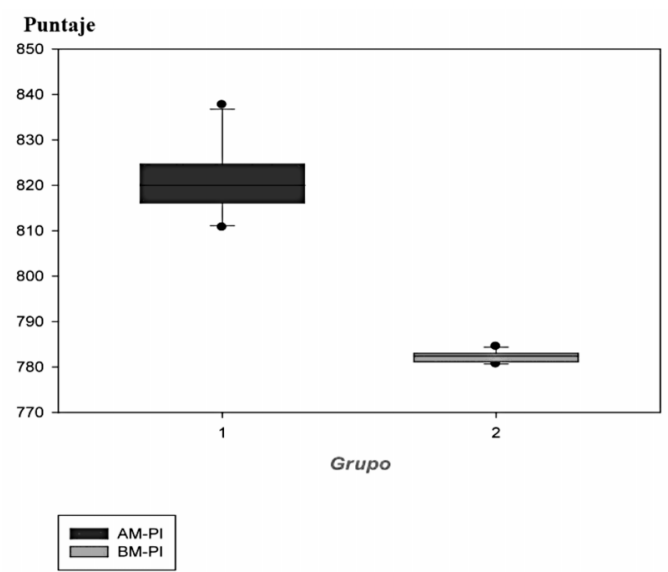

Fig. 1. Distribución de los más altos (AM-PI) y más bajos (BM-PI) puntajes de ingreso a la carrera de Medicina de la Pontificia Universidad Católica de Chile año 2009.

AM-PI: Grupo de Puntaje de ingreso alto; BM-PI: Grupo de Puntaje de ingreso bajo. Medicina 2009
Tabla IV Correlación estadística de ambos grupos muestrales para la distribución de los datos observados en el Fig. 3, con Mann-Whitney Rank Sum Test y t-test para la Prueba Global Inicial y Nota Final respectivamente.

\begin{tabular}{llllll}
\hline Group Name & $\mathbf{n}$ & Missing & Median & $\mathbf{2 5 \%}$ & $\mathbf{7 5 \%}$ \\
\hline AM-GLOBI & 10 & 0 & 5,915 & 5,700 & 6,160 \\
BM-GLOBI & 10 & 0 & 5,335 & 4,710 & 5,470 \\
Mann-Whitney & U Statistic & $T=134,000$ & $n$ (small)= 10 & $n($ big) $=10$ \\
& & & & & \\
\hline Group Name & $\mathbf{n}$ & Missing & Mean & Std & SEM \\
\hline AM-NF & 10 & 0 & 6,070 & 0,358 & 0,113 \\
BM-NF & 10 & 0 & 5,529 & 0,731 & 0,231 \\
$t$ - test & $t=2,102$ & with 18 degrees of freedom. \\
\hline
\end{tabular}

$(\mathrm{P}=<0,05)$. AM-GLOBI: Prueba Global Inicial primeros seleccionados; BM-GLOBI: Prueba Global Inicial últimos seleccionados; AM-NF: Nota Final del curso primeros seleccionados: BM- NF: Nota Final del curso últimos seleccionados. 


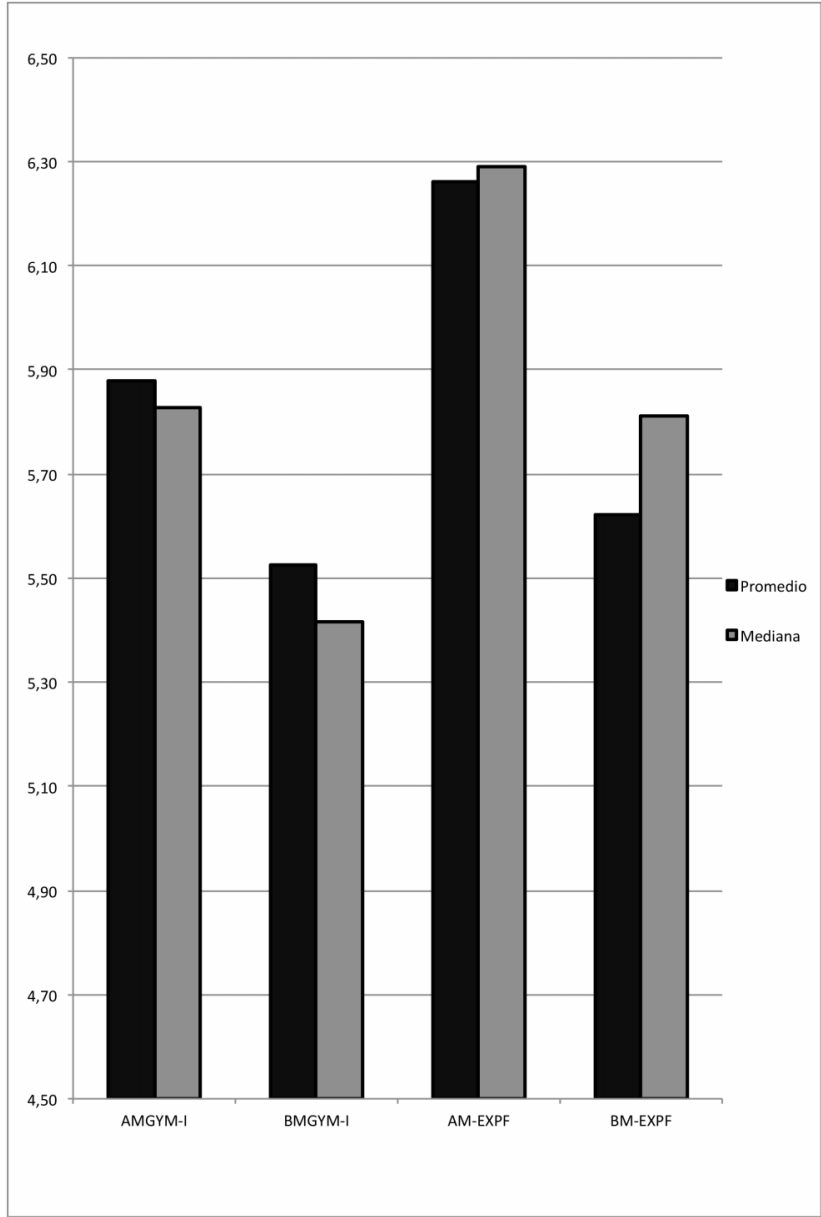

Fig. 2. Distribución de valores obtenidos en el análisis estadístico descriptivo de la muestra correspondiente a la Gymcana Inicial y Examen práctico Final por grupo.

AM-GYMI: Nota obtenida en la Gymkana Inicial por los primeros seleccionados; BM-GYMI: Nota obtenida en la Gymkana Inicial por los últimos seleccionados; AM-EXPF: Nota obtenida en el Examen Práctico Final por los primeros seleccionados; BM- EXPF: Nota obtenida en el Examen Práctico Final por los últimos seleccionados. Medicina 2009

El análisis estadístico inferencial de la muestra (n), con la prueba paramétrica de t-test y no paramétrica de MannWhitney Rank Sum Test, donde comparamos la variable nota entre los grupos de alto puntaje de ingreso (AM) y bajo puntaje de ingreso (BM), arrojan semejanzas para un método de evaluación y diferencias para otro distribuyéndose de la siguiente manera:

1. No se presentan diferencias estadísticamente significativas, entre ambos grupos, para las notas obtenidas en pruebas prácticas como la Gymkana inicial (GYMI) y el Exámen práctico final del curso (EXPF), ambos análisis con un índice de confianza de un $95 \%$; dando un valor $\mathrm{P}=0,300$ y $\mathrm{P}=$ 0,129 respectivamente. (Fig. 2, Tabla III)

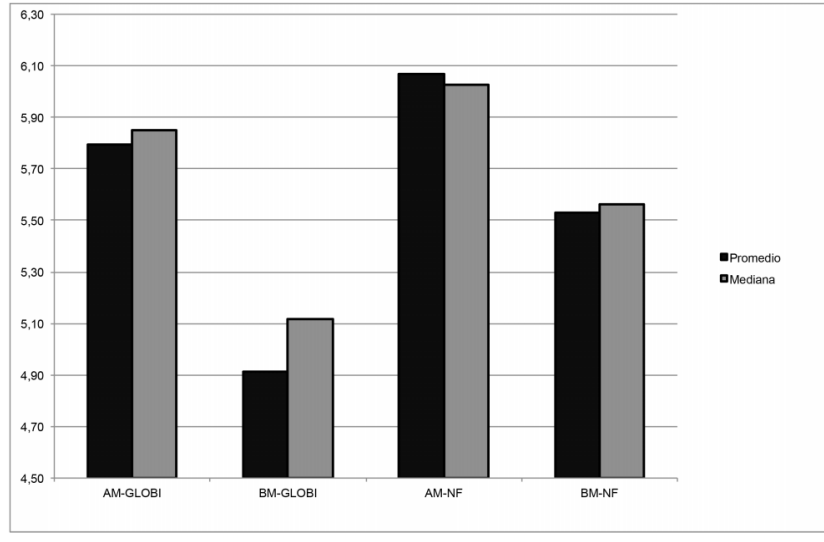

Fig. 3. Distribución de valores obtenidos en el análisis estadístico descriptivo de la muestra correspondiente a la Prueba Global Inicial y Nota Final por grupo.

AM-GLOBI: Nota obtenida en la Prueba Global Inicial por los primeros seleccionados; BM-GLOBI: Nota obtenida en la Prueba Global Inicial por los últimos seleccionados; AM-NF: Nota Final del curso obtenida por los primeros seleccionados; BM- NF: Nota Final del curso obtenida por los últimos seleccionado.

2. En cambio, sí se observan diferencias estadísticamente significativas, entre ambos grupos, para las notas obtenidas tanto, en la Global inicial (GLOBI) como en la Nota final del curso (NF), ambos análisis con un índice de confianza de un $95 \%$; dando un valor $\mathrm{P}=0,028$ y $\mathrm{P}=0,049$ respectivamente. (Fig. 3, Tabla IV)

\section{DISCUSIÓN}

El interés de ingresar a la educación superior por parte de los alumnos de diferentes establecimientos educacionales públicos y privados, ha ido incrementándose considerablemente posterior a la implementación de la PSU. El año 2004 , el 61,4 \% de la población estudiantil de cuarto año medio la rindieron; subiendo a 70,1\% este porcentaje en el año 2006 (Castillo, 2007); y llegando al 86,5 \% para el proceso de admisión 2009 (Castillo \& Cabezas, 2010).

Donoso \& Contreras (2009) analizan la confiabilidad de la prueba de selección universitaria del 2009, señalando que todas las pruebas, independiente de las variables Región, Género, Origen y Año de Egreso, presentan coeficientes de confiabilidad (a) iguales o superiores a 0,91 ; valor que, de acuerdo a estándares internacionales y nacionales, es considerado muy satisfactorio, por cuanto indica que el $91 \%$ o más de la varianza de los puntajes es producto de las diferencias individuales en rendimiento que presentan los postulantes. Este resultado asegura que la ordenación que 
se hace a partir de los puntajes es muy precisa, lo que, a su vez, avala la calidad de la selección que se lleva a cabo, que es el fin último del proceso. Es así como el puntaje determinado por una prueba de selección de Lenguaje y Comunicación, Matemáticas y otra de Ciencias (perfil humanista y científico), muestra diferencias significativas en nuestros alumnos de medicina para las pruebas de Matemáticas $(\mathrm{P}=$ $0,001)$ y ciencias $(P=0,002)$, característica que, de acuerdo al perfil del profesional médico que buscamos obtener, es la que debemos potenciar y equiparar en nuestros alumnos.

Analizando los resultados de nuestros alumnos podemos afirmar que el único tipo de evaluación niveladora, es decir que logra superar las diferencias de origen de los examinados, lo constituyen nuestras pruebas prácticas (gymkanas); evaluaciones en las cuales el rendimiento de los primeros y de los últimos seleccionados de la carrera medicina tiende a ser más homogéneo. Este tipo de evaluaciones prácticas, realizadas sobre preparaciones cadavéricas, demandan fundamentalmente experiencias vividas en nuestros pasos prácticos, además de claves visuales de calidad que son relevantes en el proceso de aprendizaje (Norbis, 1971; Inzunza \& Bravo, 2002).

A este respecto el trabajo en el laboratorio con grupos pequeños, es eficaz en la enseñanza de la anatomía humana, especialmente cuando el uso de muestras es variada, combinando preparaciones, plastinados, cadáveres e imagenología (Chan \& Ganguly, 2008), material con que cuenta nuestro departamento de anatomía y que usamos profusamente en nuestras actividades prácticas. La formación del médico debe ser un continuo que nace desde las ciencias básicas, por lo cual como anatomistas no podemos quedar fuera de los cambios curriculares internacionales, donde se busca una integración de conceptos y que los alumnos aprendan construyendo su propio conocimiento en el laboratorio, trabajando en grupos pequeños, tomando como centro el cadáver, logrando así una gran interacción profesor-alumno (Drake, 1998). Sabido es que los alumnos de medicina se interesan por hechos verificables que evalúan y usan de manera objetiva e imparcial (Bitran et al., 2003). En este contexto, la actividad práctica en Anatomía, utilizando el cadaver humano como centro, constituye la única oportunidad que tiene el médico en ciernes para confirmar empíricamente la situación de órganos y sistemas corporales y su relación con la enfermedad y la muerte. (Inzunza, 2008)

Entonces, estas actividades prácticas, utilizando material cadavérico y las evaluaciones prácticas objetivadas estructuradas (gymkanas), influyen nivelando académicamente a nuestros alumnos (Inzunza \& Salgado, 2011), independiente de la experiencia menor o mayor que traiga el estudiante de su respectivo establecimiento educacional y el capital cultural inicial que posea. De manera similar es esperable que las evaluaciones clínicas de objetivos estructurados (ECOE), sea un instrumento evaluativo que ofrezca una equidad en los temas que el alumno debe resolver y en el tiempo que dispone para este efecto.

Tal es el grado de convencimiento que tenemos sobre la incidencia positiva de las evaluaciones prácticas como elemento que permite superar las diferencias de origen que traen nuestros alumnos, que a partir del año 2014 estas evaluaciones (gymkanas) tienen un peso del $55 \%$ en la nota final del curso de Anatomía Normal.

A la luz de lo expuesto, resulta interesante considerar la evidencia internacional reciente que señala que el rendimiento en los cursos de anatomía constituye un excelente predictor en relación con el desempeño en el examen de licencia médica, el equivalente a nuestro examen médico nacional (Peterson \& Tucker, 2005; Tucker, 2008); todo ello avalado por una metodología de enseñanza en la cual se desarrollan en los estudiantes las habilidades cognitivas necesarias, utilizando un aprendizaje exploratorio donde puedan correlacionar la anatomía con diagnósticos clínicos. Por esto, es importante considerar aquellos contenidos clínicamente relevantes y que les sean útiles para la introducción temprana de esta habilidades prácticas en el pabellón de anatomía (Philip et al., 2008).

\section{DEDICATORIA}

Dedicamos este trabajo a nuestro querido co-autor, el Dr. Mario Cantín López, cuya temprana partida nos ha golpeado duramente. La Anatomía ha perdido a un destacado docente, la UFRO a un brillante investigador y nosotros a un entrañable y querido amigo.

INZUNZA, O.; SCHNEEBERGER, D.; SALGADO, G. \& CANTÍN, M. Practical activities in anatomy: leveling factor for the origin-related contrasts among medical students. Int. J. Morphol., 35(2):494-499, 2017.

SUMMARY: Level of educational achievement and screening tests for admission to higher education are strongly correlated with socioeconomic background and the type of school of the applicant, these situations would be a performance predictor for college freshmen students. Whereas the scores to be accepted into the medical career have a significant difference between the first and last selected, we wonder if there is a leveling factor that reduces the gap of origin. We analyze the performance in practical assessments and theory tests at the beginning and end of the 
academic year 2009 comparing the first and last selected according to the PSU score admission to medical studies at the Pontificia Universidad Catolica de Chile. Significant differences were observed in the performance of the first assessment of the theory of Anatomy and the final score obtained in the course, however, no significant differences in both the first practical test and practical final exam of the course. Our results suggest that the only evaluation level, which overcomes the differences of origin of the examined, are our practical tests; assessments in which the performance of the first and the last selected medical student is more homogenous. This type of practical assessments, performed on cadavers, primarily demanded experiences in our practical segment, standing by on the degree of skill or experience held by the students related to their educational institution of origin.

KEY WORDS: Medical education; Medical students; Medical learning; Ecoe; Osce.

\section{REFERENCIAS BLIOGRÁFICAS}

Barrington, E. Teaching to student diversity in higher education: How multiple intelligence theory can help. Teach. High. Educ., 9(4):421-34, 2004.

Bitran, C. M.; Zúñiga, P. D.; Lafuente, G. M.; Viviani, G. P. \& Mena, C. B. Tipos psicológicos y estilos de aprendizaje de los estudiantes que ingresan a Medicina en la Pontificia Universidad Católica de Chile. Rev. Med. Chile, 131(9):1067-78, 2003.

Castillo, J. \& Cabezas, G. Caracterización de jóvenes primera generación en educación superior. Nuevas trayectorias hacia la equidad educativa. Rev. Calid. Educ., (32):44-76, 2010.

Castillo, J. Demandas juveniles a la educación: la necesidad de fortalecer las trayectorias educativas en el sistema escolar. Rev. Obs. Juv., 16:5565, 2007.

Chacón, M. Calidad y Equidad en la Educación Media. Santiago de Chile, Biblioteca del Congreso Nacional de Chile. Departamento de Estudios, Extensión y Publicaciones. DEPESEX/BCN/Serie Informes Año XIV, $\mathrm{N}^{\circ} 129,2003$.

Chan, L. K. \& Ganguly, P. K. Evaluation of small-group teaching in human gross anatomy in a Caribbean medical school. Anat. Sci. Educ., 1(1):1922, 2008.

Contreras, D.; Gallegos, S. \& Meneses, F. Determinantes de desempeño universitario. ¿Importa la habilidad relativa? Rev. Calid. Educ., (30):1848, 2009.

Donoso, G. \& Contreras, P. Estudio de la Confiabilidad de las Pruebas de Selección Universitaria. Santiago de Chile, Documento de Trabajo $\mathrm{N}^{\circ} 01 /$ 09. Unidad de Estudios e Investigación, DEMRE, Universidad de Chile, 2009.

Drake, R. L. Anatomy education in a changing medical curriculum. Anat. Rec., 253(1):28-31, 1998.

Inzunza, H. O. \& Salgado, A. G. Objetive structured practical test in anatomy. differences in the score of questions realized in models, anatomical preparations and cadavers. Int. J. Morphol., 29(2):490-5, 2011.

Inzunza, H. O. Competencias generales en medicina, Rol de la anatomía. Int. J. Morphol., 26(2):243-6, 2008.

Inzunza, O. \& Bravo, H. Animación computacional de fotografías, un real aporte al aprendizaje práctico de anatomía humana. Rev. Chile. Anat., 20(2):151, 2002.

Inzunza, O. Morphology, the cew challenges for 2015. Int. J. Morphol., 32(3):789-93, 2014.
Norbis, G. Didáctica y Estructura de los Medios Audiovisuales. Buenos Aires, Editorial Kapelusz, 1971.

Northedge, A. Rethinking teaching in the context of diversity. Teach. High. Educ., 8(1):17-32, 2003.

Peterson, C. A. \& Tucker, R. P. Medical gross anatomy as a predictor of performance on the USMLE Step 1. Anat. Rec. B New Anat., 283(1):5$8,2005$.

Philip, C. T.; Unruh, K. P.; Lachman, N. \& Pawlina, W. An explorative learning approach to teaching clinical anatomy using student generated content. Anat. Sci. Educ., 1(3):106-10, 2008.

Rocha, P. F.; Acevedo, P. C. G. \& Flores, O. M. Perfi 1 de ingreso de los estudiantes de la Carrera de Tecnología Médica de la Universidad de Concepción (cohortes 2006 al 2008). Determinación de variables predictivas del rendimiento académico en asignaturas de ciencias. Rev. Educ. Cienc. Salud, 6(1):28-33, 2009.

Scharager, J. \& Sebastián, C. Diversidad y educación superior: algunas reflexiones iniciales. Rev. Calid. Educ., (26):17-36, 2007.

Tucker, R. P. Performance in a prematriculation gross anatomy course as a predictor of performance in medical school. Anat. Sci. Educ., 1(5):2247, 2008.

Zúñiga, D.; Mena, B.; Oliva, R.; Pedrals, N.; Padilla, O. \& Bitran, M. Modelos de predicción del rendimiento académico de los estudiantes de medicina en el ciclo básico y preclínico. Un estudio longitudinal. Rev. Med. Chile, 137(10):1291-300, 2009.

Dirección para correspondencia:

Dr. Oscar Inzunza

Alameda Bernardo O'Higgins 340

Escuela de Medicina, $7^{\circ}$ piso

Departamento de Anatomía

Santiago

CHILE

E-mail: oinzunza@med.puc.cl

Recibido : 20-03-2017

Aceptado: 30-03-2017 